\title{
Evaluation of Acute Coronary Syndrome and Cardiovascular Risk Factors in Women of Reproductive Age in Northwest Iran
}

\author{
Razieh Parizad, Maryam Chenaghlou*, Hossein Namdar
}

\begin{abstract}
Objectives: Acute Coronary Syndrome (ACS) is rare in women of reproductive age; however with increase in risk factors of Coronary Artery Disease (CAD) in this age group, the possibility of ACS in young women is expected to rise and become a significant problem in future. It seems that pattern of risk factors and disease course may be different in this group. This study was conducted to determine the status of ACS in women of reproductive age.

Materials and Methods: This is a descriptive study of women aged between 15-45 years who were admitted with ACS during the past five years from 21 March 2008 until 20 March 2013. The definition of ACS was designed to be compatible with current guidelines. Statistical analyses were performed with SPSS version 17.0.

Results: Women of reproductive age who were admitted with ACS diagnosis were $1.16 \%$ of all ACS cases. Significant number of patients (22 patients who are $42 \%$ of all patients) had atypical chest pain and 17 (32\%) had unusual symptoms. Unstable Angina (UA) was the most common type of ACS. Most of the patients had one or more CAD risk factors. Percutaneous Coronary Intervention (PCI) or Coronary Artery Bypass Graft Surgery (CABG) was performed in 25(42\%) of the female patients with documented ACS. In recent years, the prevalence of CAD has increased considerably in in this specific group.

Conclusion: ACS is rare in women of reproductive age but may occur. The clinical presentation of the women of this study is compatible to other similar investigations.

Keywords: Coronary Artery Disease, Reproductive Age, Women
\end{abstract}

\section{Introduction}

Cardiovascular disease is the leading cause of death among women, regardless of race or ethnicity. For Coronary Heart Disease (CHD) specifically, mortality rates fell in both men and women by 44\% from 2000 to 2002 . Approximately $47 \%$ of this decrease was due to the influence of evidence-based medicine [post-myocardial infarction (MI) secondary prevention, initial treatment of Acute Coronary Syndrome (ACS), heart failure treatment, revascularization for chronic angina, and use of antihypertensive and lipid-lowering therapy] and approximately $44 \%$ to a reduction in several risk factors (hypertension, dyslipidemia, smoking, and low physical activity ) in the general population (1). Although overall mortality among women decreased, mortality among young women (between 35 and 44 years of age) increased by $1.3 \%$ per year from 1997 to 2002 (2).

Whether there are fundamental differences between men and women in mortality after MI, or whether such observed differences reflect corresponding variations in baseline characteristics, has long been a topic of discussion. In addition to differences in patient related factors, studies suggest that mortality may differ among women and men according to the type of ACS at presentation (3). Incidence of ACS in women of reproductive age is reported to be low (25-57 per 100,000 per year) $(4,5)$. Rates of CHD are consistently lower in women as compared to men. The established risk factors of elevated blood pressure, high cholesterol levels and cigarette smoking appear to increase the risk of CHD in women in the same manner as in men. At younger ages lower levels of risk factors could explain the sex difference observed in CHD. In the past cigarette smoking was considerably lower in women as compared to men; however recent cohorts have shown the reversing of this trend. Thus with increase in the prevalence of cigarette smoking in women, some of the evident protection that women may have from $\mathrm{CHD}$ decreases considerably and even exceeds that in men.

CHD is the leading cause of death in women in industrialized countries and preventive interventions need to be targeted at women as well as men. The huge international variation in mortality and incidence rates in women, together with time trends and findings from migrant studies, show that a significant percentage of CHD in women 
is preventable. While there has been some debate as to whether the same lifestyle preventive advice given to men should apply to women, the rates in women closely correlate with rates in men. Thus environmental and lifestyle factors lead to high CHD rates both in men and women. Consequently population based approaches which aim at changing lifestyle factors such as diet, smoking and physical activity are likely to be beneficial in both men and women. Studies have shown over six-fold increase in the $\mathrm{CHD}$ rate of women living in countries with high CHD prevalence in comparison to men living in countries with low CHD rates, indicating that environmental factors outweigh the impact of any biologic differences existing between men and women in the susceptibility of CHD.

A key priority for research is to elucidate further the major environmental etiologic factors that determine high CHD incidence and mortality in populations. In the interim, there is abundant evidence to support measures that lead to reduction in overall levels of CHD risk factors in both women and men in the population through dietary changes such as increasing fruit and vegetable intake, reduction of saturated fat intake, increased physical activity and, most notably, stopping cigarette smoking (6).

Thus considering the above concerns it is essential to increase our knowledge of ACS in younger women, particularly those in the reproductive age group. In this study we evaluated the prevalence and demographic features of young women with ACS.

\section{Materials and Methods}

This is a retrospective analysis of all cases of ACS recorded in Shahid Madani Hospital during five years from 21 March 2008 until 20 March 2013. This registry was designed to be compatible with current ACS guidelines. The registry records any patient aged 15 and above who is diagnosed with ACS including ST-Elevation Myocardial Infarction (STEMI), Non-ST Elevation MI (NSTEMI) and UA. UA is defined as angina pectoris (or equivalent type of ischemic discomfort) with at least one of three features:

1. Being new onset and severe

2. Occurring at rest or with minimal exertion

3. Occurring with a crescendo pattern

In the presence of at least two of the following, the diagnosis of MI was made:

1. Characteristic symptoms

2. Electrocardiographic changes

3. Typical rise and fall in biochemical markers including cardiac troponins and CK-MB.

From 8717 cases of ACS over five-year period, 101 patients were women of reproductive age (taken as women between 15-45 years). The demographic features of these women including age, education, marital status, number of children( if they had), past medical history, drug history, Coronary Artery Disease (CAD) risk factors [hypertension, Diabetes mellitus, hyperlipidemia, smoking, positive family history for Ischemic Heart Disease (IHD)], time between beginning of symptoms to receiving medical care were evaluated.

\section{Results}

All statistical analyses were performed with SPSS version 17.0. From a total of 8717 patients, 5867 (67\%) were men and 2848 (32\%) were women. In the women's group 101 patients were between 15 to 45 years, including $1.16 \%$ of all the patients and $3.5 \%$ of all women with ACS diagnosis. In the men's group $430(4.93 \%)$ cases were in the same age range. $42 \%$ of the patients had atypical chest pain or unusual forms of clinical presentation such as dizziness, nausea, palpitation, weakness, headache, fatigue and fever. Median time from beginning of the symptoms to obtaining medical care was 91 hours. In 48 cases of 101 patients, the diagnosis of ACS was ruled out, and data of remainder of the cases (53 cases) was analyzed. Among the remainders 26 (49\%) had UA, 11 (20.7\%) had NSTEMI and $16(30 \%)$ had STEMI (Figure 1). Among patients with STEMI Antero lateral MI was the most common type (5.6\%), followed by inferior (3.7\%) and extensive anterior MI (1.8\%), respectively (Figure 2 ). The mean age of patients was 39.5 years (Figure 3), 51 (96.2\%)were married and most of them $(80 \%)$ had 2 children or more.

Forty one $(77.3 \%)$ did not have any university education (Table 1). 46 (86.7\%) patients had one or more CAD risk factors (Figure 4). Hypertension was observed in 34 (64.1\%), diabetes mellitus in 18 (33.9\%), HLP in 17 (32\%) and $16(30.1 \%)$ patients were smoker. Approximately 18 (33.9\%) patients had positive family history for IHD, 9 (16.9\%) had documented CAD in their past medical history and $40(75.4 \%)$ had one or more cardiac drugs in their medications. Only one case had used Oral Contra-

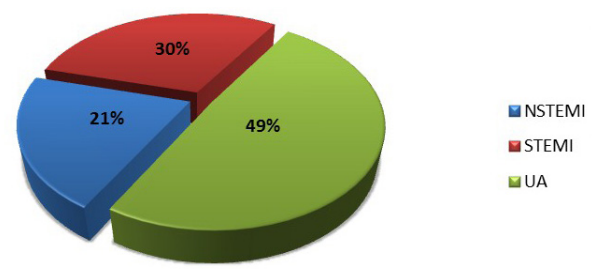

Figure 1. Types of ACS. N-STEMI: MI without ST segment elevation; STEMI: MI with ST segment elevation; UA: Unstable Angina. Data presented in percentage.

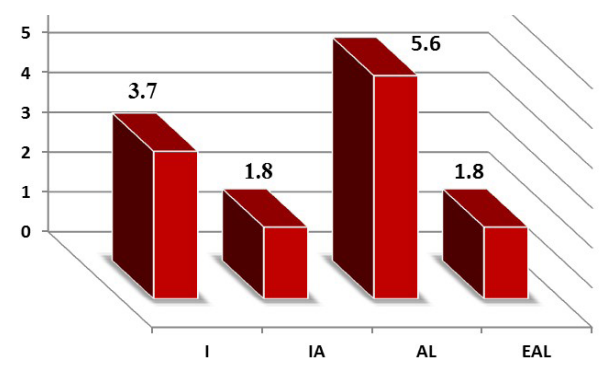

Figure 2. Location of MI. IMI: Inferior MI; IA: Inferior Anterior MI; AL: Anterior Lateral MI; EAL: Extensive Anterior Lateral MI. Data presented in percentage. 
ceptive Pills (OCP). 7 (13.2\%) patients did not have any documented CAD risk factors (Table 1).

$12(22.6 \%)$ patients had used psychological drugs alone or in combination with cardiac drugs. Medical therapy as the main stem treatment was the most common approach in $28(52.7 \%)$, while PCI was done in $16(30.18 \%)$ and CABG in $9(16.9 \%)$ of the patients (Table 2$)$. No mortality or major morbidity had been recorded. In a longitudinal evaluation, the number of patients had a significantly increasing pattern during five years (Figure 5).

\section{Discussion}

Coronary artery disease is uncommon in women of child bearing age but may occur particularly in the setting of diabetes and tobacco abuse. Acute myocardial infarction

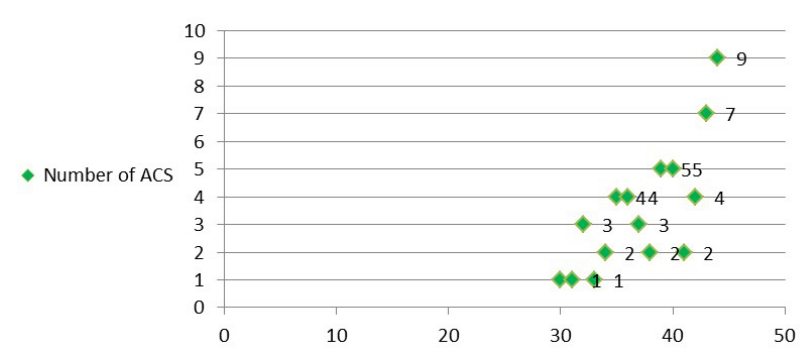

Figure 3. Number of ACS by age. Data presented by number.

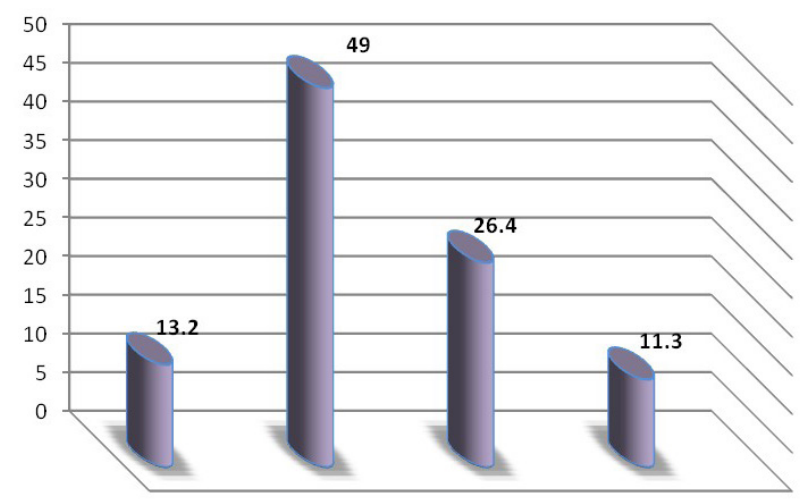

Figure 4. Number of risk factors. 1: Without risk factor; 2: One risk factor; 3: Two risk factors; 4 . Three risk factors. Data presented in percentage.

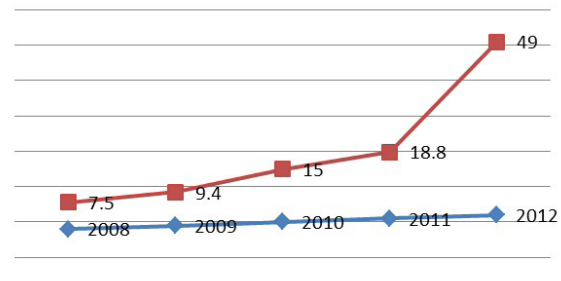

Figure 5. ACS presented by year. Data presented in percentage.
Table 1. Baseline personal characteristics and risk factors of women with ACS

\begin{tabular}{llcc}
\hline Characteristics & Number & $\%$ \\
\hline \multirow{3}{*}{ Educational } & Illiterate & 5 & 9.4 \\
& Primary education & 41 & 77.3 \\
& Diploma & 3 & 5.6 \\
& University & 4 & 7.5 \\
\hline \multirow{5}{*}{ Risk factors } & One or more CAD factors & 46 & 86.7 \\
& Hypertension & 34 & 64 \\
& Diabetes Mellitus & 18 & 33.9 \\
& Hyperlipidemia & 17 & 32 \\
& Smoking & 16 & 30 \\
& Positive family history & 18 & 33.9 \\
& Documented CAD in past & 9 & 16.9 \\
& medical history & 1 & 1.8 \\
\hline
\end{tabular}

Table 2. Final results of hospitalization

\begin{tabular}{llll}
\hline & PCI & CABG & MFU \\
\hline UA & 13.2 & 9.4 & 26.4 \\
NSTEMI & 7.5 & 1.8 & 11.3 \\
STEM & 9.4 & 5.6 & 15 \\
\hline
\end{tabular}

PCI: Percutaneous coronary intervention; CABG: Coronary artery bypass surgery; MFU: Medical follow up. Data presented in percentage.

is rare in this age group (7).

Among patients with ACS, women present more often with UA representing 30 to $45 \%$ of patients with this condition, compared with $25-30 \%$ of patients with NSTEMI and only $20 \%$ of patients of STEMI (8). In our study women of reproductive age were only $1 \%$ of total ACS patients and the most common presentation form was UA/ NSTEMI.

A study of 127 men and 90 women by Kudenchuk et al. (9) showed that among patient who presented to the emergency department with symptoms of coronary disease other than chest pain, there were several sex-related differences in the symptoms. Dyspnea, nausea and vomiting, indigestion, fatigue, sweating and arm or shoulder pain presenting in the absence of chest pain were all more frequent among women than men but the Myocardial Infarction Triage and Intervention (MITI) project investigators demonstrated that chest pain was present in almost all women (99.6\%) and men (99\%) who experienced a documented acute MI (10).

In our study $22(42 \%)$ of patients had atypical chest pain, $17(32 \%)$ had unusual presentation but chest pain was present in all women with STEMI. One of the factors related to the patient that correlate with a longer time to seek medical attention is female sex $(11,12)$. In our study this period was 91 hours which was a long time.

Primary finding of the large case control INTER HEART study provides some of the best data to date on the relation of clinical parameters with the risk of IHD worldwide, including patterns of association of MI risk among 
women compared with men. Overall, after adjustment, nine risk factors accounted for $90.4 \%$ of the population attributable risk for MI ( $94 \%$ of the population attributable risk among women and 90\% among men). The risk factor were apolipoprotein B/apolipoprotein A-I ratio, cigarette smoking, hypertension, diabetes, abdominal obesity, psychosocial factors (an index based on combining parameter estimates for depression, stress at work or home, financial stress, one or more life events, and locus of control scores), fruit and vegetable intake, exercise and alcohol intake. Although the strength of association of most risk factors with MI was similar among women and men, the INTER HEART study confirmed a markedly stronger association of diabetes with MI among women (13). Psychosocial factors also tended to associate more strongly with increased risk among women, although the difference was less in magnitude.

In our study $46(86.7 \%)$ patients had one or more CAD risk factors including hypertension, diabetes mellitus, hyperlipidemia, smoking and positive family history for IHD but the apolipoprotein $\mathrm{B}$ /apolipoprotein A ratio, amount of exercise, fruit and vegetable intake, and psychological index were not evaluated. None of the patients used alcohol. Although psychological problems were not exactly evaluated, $32 \%$ of patients had used psychological drugs which seems to be attributable to some degree of psychological problems in these patients. $13.2 \%$ of the patients did not have any recorded CAD risk factors which could be attributable to defective history taking or unevaluated risks and eventually related to some risk factors that had not been known until yet.

The STEMI guidelines are silent on sex specific recommendations, providing completely sex-neutral treatment recommendations. The UA/NSTEMI guideline provides the following class I summary recommendation regarding the treatment of women: "women with UA/NSTEMI should be managed in a manner similar to men. Specifically, women, like men with UA/NSTEMI, should receive aspirin and clopidogrel. Indications for noninvasive and invasive testing are similar in women and men (14)".

In our study 25 (47\%) patients underwent PCI or CABG. It seems that sex itself had not influenced the management and the rate of invasive strategy was acceptable.

There are several limitations in our study. Firstly this is a retrospective analysis and our data collection was based on past recordings and thus some risk factors such as psychological factors were not evaluated. Secondly the number of subjects in the study group was small.

\section{Conclusion}

Although ACS is a rare event in women of reproductive age all physicians should be aware that small percentage of women of this age, especially those with CAD risk factors could suffer from this problem and unfortunately the incidence of CAD has increased in this group of women in recent years.

\section{Ethical issues}

Written informed consent was obtained from the patients for publication of this study. The study has been approved by the local ethics committee.

\section{Conflict of interests}

Authors declare that there is no conflict of interest.

\section{Acknowledgments}

We would like to offer our gratitude to Mr. Afsari, Mrs. Habashi, Mrs. Mansoori and Mrs. Karimi from the Medical records and IT units of Shahid Madani Hospital who helped us in data collection and also our colleagues from Tabriz University of Medical Sciences and Hospital managers who supported this research.

\section{References}

1. Ford ES, Capewell S. Coronary heart disease mortality among young adults in the USA from 1980 through 2002: concealed leveling of mortality rates. J Am Coll Cardiol 2007 27;50(22):2128-32.

2. Jneid H, Fonarow GC, Cannon CP, Hernandez AF, Palacios IF, Maree AO, et al. Sex differences in medical care and early death after acute myocardial infarction. Circulation 2008;118(25):2803-10.

3. Nielsen KM, Foldspang A, Larsen ML, Gerdes LU, Rasmussen S, Faergeman O. Estimating the incidence of the acute coronary syndrome: data from a Danish cohort of 138290 persons. Eur J Cardiovascular Prev Rehabil 2007;14(5):608-14.

4. Petitti DB, Sidney S, Quesenberry CP Jr, Bernstein A. Incidence of stroke and myocardial infarction in women of reproductive age. Stroke 1997;28(2):280-3.

5. Mokdad AH, Serdula MK, Dietz WH, Bowman BA, Marks JS, Koplan JP. The spread of the obesity epidemic in the United States, 1991-1998. JAMA 1999;282(16):1519-2.

6. Wenger NK, Collins P. Women and heart disease. 2nd ed. London: Taylor \& Francis; 2005. p.14-15.

7. Roth A, Elkayam U. Acute myocardial infarction associated with pregnancy. J Am Coll Cardiol 2008;52(3):171-80.

8. Hochman JS, Tamis JE, Thompson TD, Weaver WD, White HD, Van de Werf F, et al. Sex, clinical presentation and outcome in patients with acute coronary syndromes. Global Use of Strategies to Open Occluded Coronary Arteries in Acute Coronary Syndrome IIb Investigators. N Eng J Med 1999;341(4):226-32.

9. Kudenchuk PJ, Maynard C, Martin JS, Wirkus M, Weaver WD. Comparison of presentation, treatment, and outcome of acute myocardial linfarction in men versus women (The Myocardial Infarction Triage and Intervention Registry). Am J Cardiol 1996;78(1):9-14.

10. Mensah GA, Hand MM, Antman EM, Ryan TJ Jr, Schriever R, Smith SC Jr. Development of systems of 
care for ST-elevation myocardial infarction patients: the patient and public perspective. Circulation 2007;116(2):e33-8.

11. Moser DK, Kimble LP, Alberts MJ, Alonzo A, Croft JB, Dracup K, et al. Reducing delay in seeking treatment by patients with acute coronary syndrome and stroke: a scientific statement from the American Heart Association Council on cardiovascular nursing and stroke council. Circulation 2006;114(2):168-82.

12. Yusuf S, Hawken S, Ounpuu S, Dans T, Avezum A, Lanas $\mathrm{F}$, et al. Effect of potentially modifiable risk factors associated with myocardial infarction in 52 countries (the INTER HEART study): case-control study. Lancet 2004;364(9438):937-52.
13. Antman EM, Anbe DT, Armstrong PW, Bates ER, Green LA, Hand M, et al. ACC/AHA guidelines for the management of patients with ST-elevation myocardial infarction. Part of the American College of Cardiology/American Heart Association Task Force on Practice (Writing Committee to revise the 1999 guidelines for the management of patients with acute myocardial infarction). J Am Coll Cardiol 2004;44(3):671-719.

14. Bonow RO, Mann DL Zipes DP, Peter L. Braunwald's Heart Disease: A Textbook of Cardiovascular Medicine. Single Volume, 9th ed. Philadelphia, USA: Elsevier; 2012. p. 1770-77. 